Proceedings of the Edinburgh Mathematical Society (2006) 49, 277-289 (C)

DOI:10.1017/S0013091504000070 Printed in the United Kingdom

\title{
PROPER COVERS OF AMPLE MONOIDS
}

\author{
JOHN FOUNTAIN ${ }^{1}$ AND GRACINDA M. S. GOMES ${ }^{2}$ \\ ${ }^{1}$ Department of Mathematics, University of York, Heslington, \\ York YO10 5DD, UK (jbf1@york.ac.uk) \\ ${ }^{2}$ Centro de Álgebra da Universidade de Lisboa, Avenida Professor Gama Pinto 2, \\ 1649-003 Lisboa, Portugal and \\ Departamento de Matemática, Faculdade de Ciências, Universidade de Lisboa, \\ 1746-016 Lisboa, Portugal (ggomes@cii.fc.ul.pt)
}

(Received 20 January 2004)

\begin{abstract}
Proper ample monoids are described by means of a certain category acted upon on both sides by a cancellative monoid. Making use of this characterization, we show that every ample monoid $S$ has a proper ample cover, which can be taken to be finite whenever $S$ is finite.
\end{abstract}

Keywords: covers of monoids; ample monoids; proper covers

2000 Mathematics subject classification: Primary 20M10

\section{Introduction}

On an inverse monoid $S$, we define a unary operation $(\cdot)^{+}$by $a^{+}=a a^{-1}$ for $a \in S$, thus making $S$ into a $(2,1,0)$-algebra. Similarly, there is a dual operation $(\cdot)^{*}$ given by $a^{*}=a^{-1} a$. Of course, $S$ also has the unary operation $(\cdot)^{-1}$, but this will not play a significant role in the paper.

A left ample monoid can now be defined to be a monoid $S$ with a unary operation $(\cdot)^{+}$such that there is a +-embedding (that is, a $(2,1,0)$-algebra embedding) of $S$ into an inverse monoid. As usual, $E(S)$ denotes the set of idempotents of $S$. It is immediate from the definition that, in a left ample monoid, the idempotents commute with each other, and so $E(S)$ is a subsemilattice of $S$.

Similarly, we say that a monoid $S$ with a unary operation $(\cdot)^{*}$ and a $*$-embedding into an inverse monoid is a right ample monoid. An ample monoid is one which is both left and right ample. We emphasize that the +-embedding and the $*$-embedding need not be into the same inverse monoid.

It is clear that every inverse monoid is an ample monoid with the operations $(\cdot)^{+}$and $(\cdot)^{*}$ defined as above. Cancellative monoids and full submonoids of inverse monoids are also ample.

On a left ample monoid $S$, there is a least congruence $\sigma$ such that $S / \sigma$ is a right cancellative monoid. We say that $S$ is left proper if for all elements $a$ and $b$ of $S$ such 
that $a^{+}=b^{+}$and $a \sigma b$, we have $a=b$. In the case of right ample monoids, there is the dual notion of right proper, and if $S$ is an ample monoid, then $S / \sigma$ is cancellative and $S$ is said to be proper if it is both left and right proper.

It is well known that an inverse monoid is proper if and only if it is $E$-unitary (see, for example, [6, Proposition 5.9.1]). Example 3 of [2] shows that the corresponding statement does not hold for ample monoids.

In [4] we used techniques of Margolis and Pin [10] to describe proper left ample monoids. In the present paper our first objective is to extend these methods so that they can be applied to proper ample monoids. To do this we consider certain small categories with actions on both the right and the left by cancellative monoids. We introduce the formal definitions in $\S 2$ and use them in $\S 3$ to give a structure theorem for proper (E-unitary) ample monoids.

Let $S$ be an ample monoid. We say that a proper ample monoid $P$ is a (proper) cover of $S$ if there is a map from $P$ onto $S$ which is simultaneously a +-homomorphism and a *-homomorphism of monoids that maps $E(P)$ isomorphically onto $E(S)$.

In [7], Lawson showed that every ample monoid $S$ has a proper ample cover $P$. This is also a consequence of a more general result of Simmons [12]. However, the constructions of Lawson and Simmons always yield an infinite monoid $P$, even when $S$ is finite. One purpose of the present paper is to show that finite ample monoids have finite proper covers. In $\S 4$, we present a proof of Lawson's result which does yield a finite cover $P$ whenever $S$ is finite. We thus provide a unified proof for the general and the finite cases. Our stronger result is crucial in [5], where we show that a bountiful finite semigroup belongs to the pseudovariety $\boldsymbol{A} \vee \boldsymbol{G}$ if and only if $\mathcal{H} \subseteq \mu$, where $\mu$ is the greatest congruence contained in the equivalence $\mathcal{H}^{*}$ (defined in $\S 2$ ).

\section{Preliminaries}

For basic notation and terminology regarding monoids and semigroups, we follow $[\mathbf{6}]$.

We start by giving an alternative characterization of ampleness.

On a monoid $S$ a relation $\mathcal{R}^{*}$ is defined by the rule $a \mathcal{R}^{*} b$ if and only if $a$ and $b$ are $\mathcal{R}$-related in an extension of $S$. The dual of $\mathcal{R}^{*}$ is $\mathcal{L}^{*}$, and $\mathcal{H}^{*}=\mathcal{R}^{*} \cap \mathcal{L}^{*}$. From [9] and $[\mathbf{1 1}]$, we have the following lemma.

Lemma 2.1. On a monoid $S$, for $a, b \in S$, the following are equivalent:

(a) $a \mathcal{R}^{*} b$;

(b) for all $x, y \in S, x a=y a$ if and only if $x b=y b$.

This condition simplifies when one element is idempotent.

Lemma 2.2. On a monoid $S$, for $a \in S$ and $e \in E(S)$, the following are equivalent:

(a) $a \mathcal{R}^{*} e$;

(b) $e a=a$ and for all $x, y \in S, x a=y a$ implies $x e=y e$. 
We recall that $\mathcal{R}^{*}$ is a left congruence and $\mathcal{R}$ is contained in $\mathcal{R}^{*}$. If $S$ is regular, then $\mathcal{R}^{*}=\mathcal{R}$. The $\mathcal{R}^{*}$-class of an element $a \in S$ is denoted by $R_{a}^{*}$. If $E(S)$ is a semilattice, then $R_{a}^{*}$ can contain at most one idempotent. When such an idempotent exists it is denoted by $a^{+}$. Similar results hold for $\mathcal{L}^{*}$, and when there is a unique idempotent in an $\mathcal{L}^{*}$-class $L_{a}^{*}$, it is denoted by $a^{*}$.

The following theorem is a consequence of [2, Proposition 1.2].

Theorem 2.3. A monoid $S$ is ample if and only if, for all $a \in S$ and $e \in E$,

(a) every $\mathcal{R}^{*}$-class $R_{a}^{*}$ contains a (unique) idempotent $a^{+}$;

$\left(\mathrm{a}^{\prime}\right)$ every $\mathcal{L}^{*}$-class $L_{a}^{*}$ contains a (unique) idempotent $a^{*}$;

(b) $a e=(a e)^{+} a$;

$\left(\mathrm{b}^{\prime}\right)$ ea $=a(e a)^{*}$;

(c) $E(S)$ is a semilattice.

From [2, Lemma 1.3] and its dual, we conclude that, on an ample monoid $S$, there is a minimum cancellative monoid congruence $\sigma$ defined by, for all $a, b \in S$,

$a \sigma b$ if and only if $a e=b e, \quad$ for some $e \in E(S)$.

It is easy to see that we also have

$a \sigma b$ if and only if $e a=e b, \quad$ for some $e \in E(S)$.

An ample monoid is said to be left (respectively, right) proper if $\sigma \cap \mathcal{R}^{*}=\iota$ (respectively, $\left.\sigma \cap \mathcal{L}^{*}=\iota\right)$. We say that it is proper if it is both left and right proper. It is clear that these definitions agree with those given in $\S 1$.

Observe that in an ample monoid any two idempotents are $\sigma$-related and $E(S)$ is contained in the identity of $S / \sigma$. Moreover, $E(S)$ is a $\sigma$-class if and only if $S$ is $E$-unitary. Also, we have that any proper ample monoid is $E$-unitary, but the converse is not true [2, Example 3].

We now extend the notion of ample to categories, as we did in $[\mathbf{3}]$ for the left ample (formerly left-type-A) case.

Let $\mathcal{C}$ be a (small) category with set of objects Obj $\mathcal{C}$ and set of morphisms Mor $\mathcal{C}$. For all $u \in \mathrm{Obj} \mathcal{C}$, the set of morphisms with domain (respectively, codomain) $u$ is denoted by $\operatorname{Mor}(u,-)$ (respectively, $\operatorname{Mor}(-, u)$ ). We use additive notation for composition of morphisms and represent the identity at an object $u$ by $0_{u}$. For each object $u$, the set $\operatorname{Mor}(u, u)$ is a monoid with identity $0_{u}$ called the local monoid at $u$.

The relation $\mathcal{R}^{*}$ is defined on $\operatorname{Mor} \mathcal{C}$ by the rule that $(p, q) \in \mathcal{R}^{*}$ if and only if, for all $s, t \in \operatorname{Mor} \mathcal{C}$,

$$
s+p=t+p \Leftrightarrow s+q=t+q
$$

where we make the convention that writing $s+p=t+p$ implies that both $s+p$ and $t+p$ are defined. Dually we define a relation $\mathcal{L}^{*}$. 
An element $p \in \operatorname{Mor} \mathcal{C}$ is said to be idempotent if $p+p$ is defined and $p=p+p$. A category is locally idempotent if every element of each local monoid is idempotent.

The following lemma is easily proved.

Lemma 2.4. Let $\mathcal{C}$ be a category, $u \in \operatorname{Obj} \mathcal{C}$ and $p, q \in \operatorname{Mor} \mathcal{C}$. Then

(a) if $p \in \operatorname{Mor}(u,-)$ and $(p, q) \in \mathcal{R}^{*}$, then $q \in \operatorname{Mor}(u,-)$;

(b) if $p$ is idempotent and $p \in \operatorname{Mor}(u, v)$, then $u=v$;

(c) if $p$ is idempotent, then $(p, q) \in \mathcal{R}^{*}$ if and only if $q=p+q$ and, for all $s, t \in \operatorname{Mor} \mathcal{C}$,

$$
s+q=t+q \Rightarrow s+p=t+p ;
$$

(d) $\mathcal{R}^{*}$ is a left congruence on the partial semigroup Mor $\mathcal{C}$.

Clearly, the dual of Lemma 2.4 holds for $\mathcal{L}^{*}$.

A category $\mathcal{C}$ is said to be ample if, for all $u \in \operatorname{Obj} \mathcal{C}$, the set $E(\operatorname{Mor}(u, u))$ forms a semilattice; each $\mathcal{R}^{*}$-class $R_{p}^{*}$ contains an idempotent $p^{+}$that is necessarily unique; each $\mathcal{L}^{*}$-class $L_{p}^{*}$ contains an idempotent $p^{*}$ that is necessarily unique; and, for all $p, q \in \operatorname{Mor} \mathcal{C}$,

$$
q+p^{+}=\left(q+p^{+}\right)^{+}+q
$$

and

$$
p^{*}+q=q+\left(p^{*}+q\right)^{*} .
$$

Thus a category is ample if and only if it is both left and right ample (or type-A) [4]. Note that each local monoid of an ample category is ample, and also that in an ample category, $\left(p^{+}\right)^{+}=p^{+}$and $\left(p^{*}\right)^{*}=p^{*}$ for each morphism $p$.

An ample category is said to be proper if, for all $p, q \in \operatorname{Mor}(u, v)$,

$$
p^{+}=q^{+} \quad \Rightarrow \quad p=q \quad \text { (left proper) }
$$

and

$$
p^{*}=q^{*} \quad \Rightarrow \quad p=q \quad \text { (right proper) } .
$$

It follows immediately from the preceding paragraph that if an ample category is proper, then it is locally idempotent, for if $p$ is in a local monoid, then $p^{+}$is in the same monoid and so $p^{+}=p$ follows from $\left(p^{+}\right)^{+}=p^{+}$.

As in [4] we say that a monoid $B$ acts on the right on a category $\mathcal{C}$ if there exist maps $\operatorname{Obj} \mathcal{C} \times B \rightarrow \operatorname{Obj} \mathcal{C}$ and $\operatorname{Mor} \mathcal{C} \times B \rightarrow \operatorname{Mor} \mathcal{C}$, where we write $x b$ for the result of the action of the monoid element $b$ on an object or morphism $x$, and the following conditions are satisfied.

(a) If $p \in \operatorname{Mor}(u, v)$, then $p b \in \operatorname{Mor}(u b, v b)$.

(b) For all $b, b_{1}, b_{2} \in B, p, q \in \operatorname{Mor} \mathcal{C}, u \in \operatorname{Obj} \mathcal{C}$ :

(i) $(p+q) b=p b+q b$;

(ii) $\left(p b_{1}\right) b_{2}=p\left(b_{1} b_{2}\right)$;

(iii) $p 1=p$;

(iv) $0_{u} b=0_{u b}$. 
When $B$ is right cancellative, we always assume that the action is right cancellative, that is, it satisfies the following conditions.

(c) For all $p, q \in \operatorname{Mor} \mathcal{C}$ and $b \in B$,

$$
p b=q b \quad \Rightarrow \quad p=q .
$$

(d) For all $u \in \operatorname{Obj} \mathcal{C}$ and $b \in B$, if $q \in \operatorname{Mor}(w, u b)$, then $w=v b$ for some object $v$ and $q=p b$ for some $p \in \operatorname{Mor}(v, u)$.

Dually, we can define the notion of a left action of a left cancellative monoid $A$ on a category $\mathcal{C}$, and we always assume that such an action is left cancellative.

If monoids $A$ and $B$ act on $\mathcal{C}$ simultaneously, we require the actions to be compatible, that is, for all $p \in \operatorname{Mor} \mathcal{C}, a \in A$ and $b \in B$,

$$
(a p) b=a(p b) .
$$

In this case we denote $(a p) b$ by $a p b$.

If $A$ and $B$ are cancellative and $\mathcal{C}$ is ample, it follows from the proof of $[\mathbf{1 2}$, Proposition 25] or is easily proved directly that, for all $p \in \operatorname{Mor} \mathcal{C}, a \in A$ and $b \in B$,

$$
(a p)^{+}=a p^{+}, \quad(a p)^{*}=a p^{*}, \quad(p b)^{*}=p^{*} b \quad \text { and } \quad(p b)^{+}=p^{+} b .
$$

An ample category $\mathcal{C}$ acted upon on the left by a cancellative monoid $A$ and on the right by a cancellative monoid $B$ is said to be an ample $(A, B)$-category if $\operatorname{Obj} \mathcal{C}=A \times B$ and, for all $(u, v) \in \operatorname{Obj} \mathcal{C}, a \in A$ and $b \in B$,

$$
a(u, v)=(a u, v) \quad \text { and } \quad(u, v) b=(u, v b) .
$$

We note that an ample $(A, B)$-category is locally idempotent if and only if $\mathcal{M}=$ $\operatorname{Mor}((1,1),(1,1))$ is a semilattice. For, if $a \in A, b \in B$ and $q \in \operatorname{Mor}((a, b),(a, b))$, then $(a, b)=a(1,1) b$, so that $q=a p b$ for some $p \in \mathcal{M}$. Hence $q+q=a p b+a p b=a(p+p) b=$ $a p b=q$.

\section{Proper ample monoids}

The approach in this section is a slight variation on that in [12], where Simmons obtains much more general results. For completeness, we give proofs of our structure theorems for ample, $E$-unitary ample and proper ample monoids. Rather than using the general notion of double semidirect product as in $[\mathbf{1 2}]$, we use the special case of the ample $(A, B)$-categories introduced at the end of the preceding section.

We start by showing how to associate a monoid $C_{(1,1)}$ to an $(A, B)$-category. Given an ample $(A, B)$-category $\mathcal{C}$, let

$$
C_{(1,1)}=\{(b, p, a): b \in B, a \in A, p \in \operatorname{Mor}((1, b),(a, 1))\}
$$

and, for $(b, p, a),(d, q, c) \in C_{(1,1)}$, define

$$
(b, p, a)(d, q, c)=(b d, p d+a q, a c) .
$$


Notice that this operation is well defined since, if $p$ and $q$ are in $\operatorname{Mor}((1, b),(a, 1))$ and $\operatorname{Mor}((1, d),(c, 1))$, respectively, then

$$
p d \in \operatorname{Mor}((1, b d),(a, d)) \text { and } a q \in \operatorname{Mor}((a, d),(a c, 1)),
$$

so that $p d+a q$ is defined and belongs to $\operatorname{Mor}((1, b d),(a c, 1))$.

Theorem 3.1. Let $A$ and $B$ be cancellative monoids. If $\mathcal{C}$ is an ample $(A, B)$-category, then $C_{(1,1)}$ is an ample monoid. Moreover, if $\mathcal{C}$ is locally idempotent, then $C_{(1,1)}$ is E-unitary, and if $\mathcal{C}$ is proper, then so is $C_{(1,1)}$.

Proof. Throughout the proof, we denote $\operatorname{Mor}((1,1),(1,1))$ by $\mathcal{M}$.

First, it is routine to show that the binary operation defined on $C_{(1,1)}$ is associative with identity element $\left(1,0_{(1,1)}, 1\right)$.

Since both $A$ and $B$ are cancellative monoids, we get

$$
E\left(C_{(1,1)}\right)=\{(1, p, 1): p \in E(\mathcal{M})\}
$$

and so it is clear that $E\left(C_{(1,1)}\right)$ is isomorphic to $E(\mathcal{M})$, which is a semilattice since $\mathcal{C}$ is ample.

Given $p \in \operatorname{Mor}((1, b),(a, 1))$ there exists a unique idempotent $p^{+}$in its $\mathcal{R}^{*}$-class and $p^{+} \in \operatorname{Mor}((1, b),(1, b))$. As the action of $B$ on $\mathcal{C}$ is right cancellative and $(1, b)=(1,1) b$, there exists a unique $p_{0}^{+} \in \mathcal{M}$ such that $p^{+}=p_{0}^{+} b$. Again by the right cancellativity of the action, $p_{0}^{+}$is also idempotent. Now

$$
\left(1, p_{0}^{+}, 1\right)(b, p, a)=\left(b, p_{0}^{+} b+p, a\right)=(b, p, a) .
$$

Suppose that, for some $\left(d_{1}, p_{1}, c_{1}\right)$ and $\left(d_{2}, p_{2}, c_{2}\right) \in \operatorname{Mor} \mathcal{C}$,

$$
\left(d_{1}, p_{1}, c_{1}\right)(b, p, a)=\left(d_{2}, p_{2}, c_{2}\right)(b, p, a) .
$$

Then

$$
\left(d_{1} b, p_{1} b+c_{1} p, c_{1} a\right)=\left(d_{2} b, p_{2} b+c_{2} p, c_{2} a\right)
$$

and so

$$
d_{1} b=d_{2} b, \quad c_{1} a=c_{2} a \quad \text { and } \quad p_{1} b+c_{1} p=p_{2} b+c_{2} p .
$$

Since $A$ and $B$ are right cancellative, $d_{1}=d_{2}$ and $c_{1}=c_{2}$. Thus $p_{1} b+c_{1} p=p_{2} b+c_{1} p$. Now

$$
\left(c_{1} p\right)^{+}=c_{1} p^{+} \quad \text { and so } \quad p_{1} b+c_{1} p^{+}=p_{2} b+c_{1} p^{+} .
$$

But $p^{+}=p_{0}^{+} b$ and the actions are compatible, so by the right cancellativity of the action of $B$, we get

$$
p_{1}+c_{1} p_{0}^{+}=p_{2}+c_{1} p_{0}^{+} \text {. }
$$

Therefore,

$$
\left(d_{1}, p_{1}, c_{1}\right)\left(1, p_{0}^{+}, 1\right)=\left(d_{2}, p_{2}, c_{2}\right)\left(1, p_{0}^{+}, 1\right)
$$

and so the idempotent $\left(1, p_{0}^{+}, 1\right)$ is $\mathcal{R}^{*}$-related to $(b, p, a)$. 
Next, for $(1, r, 1) \in E\left(C_{(1,1)}\right)$ and $(b, p, a) \in C_{(1,1)}$, we have

$$
\begin{aligned}
((b, p, a)(1, r, 1))^{+}(b, p, a) & =(b, p+a r, a)^{+}(b, p, a) \\
& =\left(1,(p+a r)_{0}^{+}, 1\right)(b, p, a) \\
& =\left(b,(p+a r)_{0}^{+} b+p, a\right) \\
& =\left(b,(p+a r)^{+}+p, a\right) \\
& =(b, p+a r, a) \\
& =(b, p, a)(1, r, 1)
\end{aligned}
$$

Thus $C_{(1,1)}$ is left ample. The proof of the dual properties for $\mathcal{L}^{*}$ is similar. We conclude that $C_{(1,1)}$ is an ample monoid.

Now suppose that $\mathcal{C}$ is locally idempotent and let $(b, p, a),(1, q, 1) \in C_{(1,1)}$ be such that $(b, p, a)(1, q, 1)$ is idempotent, that is, $(b, p+a q, a)$ is idempotent. Then $b=1$ and $a=1$, so that $p$ is in $\mathcal{M}$. By assumption, $p$ is idempotent and hence so is $(b, p, a)$. Thus $C_{(1,1)}$ is E-unitary.

It remains to show that $C_{(1,1)}$ is proper whenever $\mathcal{C}$ is proper. Suppose that $\mathcal{C}$ is proper and that $(b, p, a),(d, q, c) \in C_{(1,1)}$ are $\sigma$-related and $\mathcal{R}^{*}$-related. Then $\left(1, p_{0}^{+}, 1\right)=$ $\left(1, q_{0}^{+}, 1\right)$, and so $p_{0}^{+}=q_{0}^{+}$.

On the other hand, there is an idempotent $(1, r, 1)$ such that

$$
(b, q, a)(1, r, 1)=(d, q, c)(1, r, 1)
$$

Thus $b=d$ and $a=c$. Hence $p, q \in \operatorname{Mor}((1, b),(a, 1))$ and $p^{+}=p_{0}^{+} b=q_{0}^{+} b=q_{0}^{+} d=q^{+}$. As $\mathcal{C}$ is left proper, $p=q$. Therefore, $\mathcal{R}^{*} \cap \sigma=\iota$ in $C_{(1,1)}$.

Similarly, we can show that $\mathcal{L}^{*} \cap \sigma=\iota$ in $C_{(1,1)}$, and so $C_{(1,1)}$ is proper.

Our next task is to prove that any (proper, $E$-unitary) ample monoid is isomorphic to a suitable monoid $C_{(1,1)}$.

Theorem 3.2. A monoid $M$ with unary operations $(\cdot)^{*}$ and $(\cdot)^{+}$is ample if and only if it is $(2,1,1,0)$-isomorphic to a monoid $C_{(1,1)}$ associated with an ample $(A, B)$-category, where $A$ and $B$ are cancellative monoids. Moreover, it is proper (E-unitary) if and only if the $(A, B)$-category is proper (locally idempotent).

Proof. In view of the previous theorem it remains to show the direct part.

Suppose that $M$ is an ample monoid. Let $\sigma$ be the least cancellative congruence on $M$ and put $A=B=M / \sigma$. We define the derived $(A, B)$-category $\mathcal{D}$ of the canonical epimorphism $\theta: M \rightarrow M / \sigma$ given by $a \theta=a \sigma$ as follows.

$\operatorname{Obj} \mathcal{D}=A \times B$ and, for $\left(a_{1}, b_{1}\right),\left(a_{2}, b_{2}\right) \in A \times B$,

$$
\operatorname{Mor}\left(\left(a_{1}, b_{1}\right),\left(a_{2}, b_{2}\right)\right)=\left\{\left(\left(a_{1}, b_{1}\right), m,\left(a_{2}, b_{2}\right)\right): a_{1}(m \theta)=a_{2}, b_{1}=(m \theta) b_{2}\right\}
$$

with composition defined by

$$
\left(\left(a_{1}, b_{1}\right), m,\left(a_{2}, b_{2}\right)\right)+\left(\left(a_{2}, b_{2}\right), n,\left(a_{3}, b_{3}\right)\right)=\left(\left(a_{1}, b_{1}\right), m n,\left(a_{3}, b_{3}\right)\right) .
$$


The action of $A$ on the left is defined as follows: for $a \in A$ and $\left(\left(a_{1}, b_{1}\right), m,\left(a_{2}, b_{2}\right)\right) \in$ Mor $\mathcal{D}$,

$$
a\left(\left(a_{1}, b_{1}\right), m,\left(a_{2}, b_{2}\right)\right)=\left(\left(a a_{1}, b_{1}\right), m,\left(a a_{2}, b_{2}\right)\right) .
$$

We define the action of $B$ on the right similarly.

It is routine to prove that the composition is well defined and associative. Given $(a, b)$ in $\operatorname{Obj} \mathcal{D}$, we have $0_{(a, b)}=\left((a, b), 1_{M},(a, b)\right)$, where $1_{M}$ is the identity of $M$.

Let $(a, b) \in \operatorname{Obj} \mathcal{D}$. Then, since $E(M)$ is a semilattice, so is

$$
E(\operatorname{Mor}((a, b),(a, b)))=\{((a, b), m,(a, b)): m \in E(M)\} .
$$

To prove that $\mathcal{D}$ is left ample is also routine after noticing that

$$
\left(\left(a_{1}, b_{1}\right), m,\left(a_{2}, b_{2}\right)\right)^{+}=\left(\left(a_{1}, b_{1}\right), m^{+},\left(a_{1}, b_{1}\right)\right),
$$

for any $\left(\left(a_{1}, b_{1}\right), m,\left(a_{2}, b_{2}\right)\right) \in \operatorname{Mor} \mathcal{D}$.

A dual argument shows that $\mathcal{D}$ is right ample, and thus $\mathcal{D}$ is ample.

To conclude that $\mathcal{D}$ is an $(A, B)$-category, it remains to analyse the properties of the actions, but these are straightforward to verify.

Next we show that $M$ and $C_{(1,1)}$ are isomorphic as (2,1,1,0)-algebras.

Observe that

$$
\begin{aligned}
C_{(1,1)} & =\{(b,((1, b), m,(a, 1)), a): a \in A, b \in B, b=m \theta=a\} \\
& =\{(m \theta,((1, m \theta), m,(m \theta, 1)), m \theta): m \in M\}
\end{aligned}
$$

and define $\psi: M \rightarrow C_{(1,1)}$ by putting

$$
m \psi=(m \theta,((1, m \theta), m,(m \theta, 1)), m \theta) .
$$

Clearly, $\psi$ is both injective and onto. To prove that $\psi$ is a monoid homomorphism is straightforward.

To show that $\psi$ respects the unary operation $(\cdot)^{+}$, we consider $m \in M$ and $p=$ $((1, m \theta), m,(m \theta, 1))$. Then

$$
\begin{aligned}
(m \psi)^{+} & =(m \theta,((1, m \theta), m,(m \theta, 1)), m \theta)^{+} \\
& =\left(1, p_{0}^{+}, 1\right),
\end{aligned}
$$

where $p^{+}=\left((1, m \theta), m^{+},(1, m \theta)\right)$ and $p_{0}^{+}=\left((1,1), m^{+},(1,1)\right)$. Hence $(m \psi)^{+}=m^{+} \psi$.

Similarly, one can show that $\psi$ respects the operation $(\cdot)^{*}$. Therefore, $\psi$ is a (2,1,1,0)-isomorphism, as required.

Suppose that $M$ is $E$-unitary. Then $E(M)$ is the identity of $M / \sigma$ and, for any object $(a, b)$ of $\mathcal{D}$,

$$
\begin{aligned}
\operatorname{Mor}((a, b),(a, b)) & =\{((a, b), m,(a, b)): m \theta=1\} \\
& =\{((a, b), m,(a, b)): m \in E(M)\} \\
& \simeq E(M) .
\end{aligned}
$$

Thus $\mathcal{D}$ is locally idempotent. 
As in $[\mathbf{4}$, Theorem 2.2], we can show that $\mathcal{D}$ is left proper (and dually that it is right proper), whenever $M$ is left (right) proper.

\section{Proper covers of ample monoids}

This section is devoted to proving that any (finite) ample monoid has a (finite) proper ample cover. We use relational morphisms to associate an ample $(A, B)$-category with an ample monoid $M$ in such a way that the proper ample monoid $C_{(1,1)}$ is a cover of $M$.

Let $M$ be an ample monoid. Recall that a (proper) ample monoid $P$ is said to be a (proper) cover of $M$ if there exists a $(2,1,1,0)$-morphism $\theta$ from $P$ onto $M$ that separates idempotents. We remark that the latter condition is equivalent to the restriction of $\theta$ to $E(P)$ being an isomorphism onto $E(M)$.

For any subset $X$ of a left ample monoid $T$, we put $X^{+}=\left\{x^{+}: x \in X\right\}$. A relational morphism $\tau: M \rightarrow T$ of left ample monoids $M$ and $T$ is a relational morphism of $(2,1,0)$-algebras, that is, $\tau$ maps $M$ to $\mathcal{P}(T)$ and, for all $m, n \in M$, we have

(1) $m \tau \neq \emptyset$,

(2) $(m \tau)^{+} \subseteq m^{+} \tau$,

(3) $(m \tau)(n \tau) \subseteq(m n) \tau$,

(4) $1_{T} \in 1_{M} \tau$.

These conditions ensure that the graph of $\tau$, which we denote by $\operatorname{gr}(\tau)$, is a $(2,1)$ subalgebra of the direct product $M \times T$, and that the projection of $\operatorname{gr}(\tau)$ to $M$ is surjective. In particular, $\operatorname{gr}(\tau)$ is a left ample submonoid of $M \times T$. We also note that if $T$ is right cancellative, then $1_{T} \in e \tau$ for every idempotent $e$ of $M$; for, e $\tau$ is not empty, and if $t \in e \tau$, then $1_{T}=t^{+} \in(e \tau)^{+} \subseteq e \tau$.

Given a relational morphism $\tau: M \rightarrow T$ from a left ample monoid $M$ to a right cancellative monoid $T$, we say that $\tau$ is left proper if, for all $m, n \in M$,

$$
m \tau \cap n \tau \neq \emptyset \quad \text { implies } n^{+} m=m^{+} n .
$$

We remark that, as noted in [1], a left proper relational morphism $\tau: M \multimap T$ is idempotent pure in the sense that if $1_{T} \in m \tau$, then $m$ is idempotent.

For a right ample monoid $M$ and a left cancellative monoid $T$, we have the dual notion of a right proper relational morphism $\tau$ and such a $\tau$ is also idempotent pure.

In the following lemma, we give an alternative characterization of a left proper relational morphism which looks more like the definition of a left proper ample category.

Lemma 4.1. A relational morphism $\tau: M \rightarrow T$ from a left ample monoid $M$ to a right cancellative monoid $T$ is left proper if and only if

$$
m \tau \cap n \tau \neq \emptyset \quad \text { and } \quad m^{+}=n^{+} \quad \text { imply } m=n .
$$


Proof. If $\tau$ is left proper, and if $m \tau \cap n \tau \neq \emptyset$ and $m^{+}=n^{+}$, then $m=m^{+} m=$ $n^{+} m=m^{+} n=n^{+} n=n$.

Conversely, suppose that the condition of the lemma holds and that $m \tau \cap n \tau \neq \emptyset$. Since $1 \in e \tau$ for any idempotent in $M$, we have

$$
m \tau \cap n \tau \subseteq\left(n^{+} \tau\right)(m \tau) \cap\left(m^{+} \tau\right)(n \tau) \subseteq\left(n^{+} m \tau\right) \cap\left(m^{+} n \tau\right)
$$

so that the latter is non-empty. Now $\left(n^{+} m\right)^{+}=m^{+} n^{+}=n^{+} m^{+}=\left(m^{+} n\right)^{+}$, and so the condition gives $n^{+} m=m^{+} n$.

Now let $M$ be an ample monoid, let $A, B$ be cancellative monoids, let $\tau_{1}: M \rightarrow A$ be a relational morphism of right ample monoids, and let $\tau_{2}: M \rightarrow B$ be a relational morphism of left ample monoids. We construct an ample $(A, B)$-category $\mathcal{X}=\mathcal{X}_{\left(\tau_{1}, \tau_{2}\right)}$. Let $\operatorname{Obj} \mathcal{X}=A \times B$. For $\left(a_{1}, b_{1}\right),\left(a_{2}, b_{2}\right) \in A \times B$,

$$
\operatorname{Mor}\left(\left(a_{1}, b_{1}\right),\left(a_{2}, b_{2}\right)\right)=\left\{\left(\left(a_{1}, b_{1}\right), m,\left(a_{2}, b_{2}\right)\right): a_{2} \in a_{1}\left(m \tau_{1}\right), b_{1} \in\left(m \tau_{2}\right) b_{2}\right\} .
$$

Composition is given as in the derived $(A, B)$-category of $\S 3$ as follows:

$$
\left(\left(a_{1}, b_{1}\right), m,\left(a_{2}, b_{2}\right)\right)+\left(\left(a_{2}, b_{2}\right), n,\left(a_{3}, b_{3}\right)\right)=\left(\left(a_{1}, b_{1}\right), m n,\left(a_{3}, b_{3}\right)\right),
$$

for $\left(\left(a_{1}, b_{1}\right), m,\left(a_{2}, b_{2}\right)\right),\left(\left(a_{2}, b_{2}\right), n,\left(a_{3}, b_{3}\right)\right) \in \operatorname{Mor} \mathcal{X}$. It is routine to check that the composition is well defined and associative. For any idempotent $e$ of $M$ and elements $a$ and $b$ of $A$ and $B$, respectively, we have $a=a 1_{A} \in a\left(e \tau_{1}\right)$ and $b=1_{B} b \in\left(e \tau_{2}\right) b$, and it follows that

$$
E(\operatorname{Mor}((a, b),(a, b)))=\{((a, b), e,(a, b)): e \in E(M)\} .
$$

It is obvious then that $E(\operatorname{Mor}((a, b),(a, b)))$ is isomorphic to the semilattice $E(M)$.

Now let $p=\left(\left(a_{1}, b_{1}\right), m,\left(a_{2}, b_{2}\right)\right) \in \operatorname{Mor} \mathcal{X}$. Then $m^{*}, m^{+} \in E(M)$ and, consequently, $\left(\left(a_{1}, b_{1}\right), m^{+},\left(a_{1}, b_{1}\right)\right)$ and $\left(\left(a_{2}, b_{2}\right), m^{*},\left(a_{2}, b_{2}\right)\right)$ are idempotents of Mor $\mathcal{X}$. The proof that the first is $\mathcal{R}^{*}$-related to $p$, and the second is $\mathcal{L}^{*}$-related to $p$, follows as in the case of the derived $(A, B)$-category $\mathcal{D}$. Also, as with $\mathcal{D}$, we prove that $\mathcal{X}$ is ample and define an action on the right (respectively, left) of $\mathcal{D}$ by $B$ (respectively, $A$ ) as follows: for all $\left(a_{1}, b_{1}\right) \in \operatorname{Obj} \mathcal{X}, a \in A, b \in B,\left(\left(a_{1}, b_{1}\right), m,\left(a_{2}, b_{2}\right)\right) \in \operatorname{Mor} \mathcal{C}$,

$$
\begin{aligned}
& \left(a_{1}, b_{1}\right) b=\left(a_{1}, b_{1} b\right) \quad \text { and } a\left(a_{1}, b_{1}\right)=\left(a a_{1}, b_{1}\right), \\
& \left(\left(a_{1}, b_{1}\right), m,\left(a_{2}, b_{2}\right)\right) b=\left(\left(a_{1}, b_{1} b\right), m,\left(a_{2}, b_{2} b\right)\right), \\
& a\left(\left(a_{1}, b_{1}\right), m,\left(a_{2}, b_{2}\right)\right)=\left(\left(a a_{1}, b_{1}\right), m,\left(a a_{2}, b_{2}\right)\right) .
\end{aligned}
$$

Notice that the actions are well defined. For example, the action of $B$ is well defined because if $b_{1} \in\left(m \tau_{2}\right) b_{2}$, then $b_{1} b \in\left(m \tau_{2}\right) b_{2} b$.

It is routine to verify the properties of the actions and we conclude that $\mathcal{X}$ is an ample $(A, B)$-category.

If $\tau_{1}$ is left proper and $\tau_{2}$ is right proper, then the $(A, B)$-category $\mathcal{X}$ is proper. To show that $\mathcal{X}$ is left proper, suppose that $p, q \in \operatorname{Mor}\left(\left(a_{1}, b_{1}\right),\left(a_{2}, b_{2}\right)\right)$ and $p^{+}=q^{+}$. Then 
$p=\left(\left(a_{1}, b_{2}\right), m,\left(a_{2}, b_{2}\right)\right)$ and $q=\left(\left(a_{1}, b_{1}\right), n,\left(a_{2}, b_{2}\right)\right)$, for some $m, n \in M$, and $m^{+}=n^{+}$. Now, $a_{2}=a_{1} x$, for some $x \in m \tau_{1}$, and $a_{2}=a_{1} y$, for some $y \in n \tau_{1}$. Since $A$ is cancellative, $x=y$ and so $m \tau_{1} \cap n \tau_{1} \neq \emptyset$. As $m^{+}=n^{+}$and $\tau_{1}$ is left proper, it follows by Lemma 4.1 that $m=n$. Thus $p=q$, as required. Similarly, $\mathcal{X}$ is right proper, and so it is proper.

Theorem 4.2. Any (finite) ample monoid has a (finite) proper ample cover.

Proof. Let $M$ be an ample monoid. Since $M$ is left ample, it follows from [2, Proposition 1.2] that there is a $(\cdot)^{+}$-embedding (of monoids) $\rho: M \rightarrow \mathcal{I}(M)$ into the symmetric inverse monoid on $M$ which maps an element $m$ of $M$ to $\rho_{m}$, where $\rho_{m}: M m^{+} \rightarrow M m$ is given by $x \rho_{m}=x m$.

Let $X=M$ if $M$ is finite, and if $M$ is infinite, let $X=M \cup N$, where $N$ is a set of the same cardinality as $M$ and is disjoint from $M$. Then $\mathcal{I}(M)$ is an inverse subsemigroup of $\mathcal{I}(X)$ and so we may regard $\rho$ as a +-embedding (of semigroups) of $M$ into $\mathcal{I}(X)$. It follows from [8, Proposition 2.1.1] that, for every element $\varphi$ of $\mathcal{I}(M)$, there is a unit (that is, a bijection) $\alpha$ of $\mathcal{I}(X)$ such that $\varphi \leqslant \alpha$, where ' $\leqslant$ ' is the natural partial order on $\mathcal{I}(X)$.

Dually, as $M$ is right ample, we have a *-embedding of semigroups $\lambda: M \rightarrow \mathcal{I}(X)^{*}$ mapping an element $m$ of $M$ to the partial bijection $\lambda_{m}: m^{*} M \rightarrow m M$ given by $\lambda_{m}(x)=m x$.

Denote by $\mathcal{G}(X)$ the group of units of $\mathcal{I}(X)$, that is, the symmetric group on $X$ with the maps written on the right of their arguments. Its left-right dual $\mathcal{G}(X)^{*}$ is the group of units of $\mathcal{I}(X)^{*}$.

Now let

$$
H_{\rho}=\left\{\alpha \in \mathcal{G}(X): \rho_{m} \leqslant \alpha \text { for some } m \in M\right\}
$$

and

$$
H_{\lambda}=\left\{\alpha \in \mathcal{G}(X)^{*}: \lambda_{m} \leqslant \alpha \text { for some } m \in M\right\} .
$$

If $\alpha, \beta \in H_{\rho}$, then $\rho_{m} \leqslant \alpha$ and $\rho_{n} \leqslant \beta$, for some $m, n \in M$. Hence $\rho_{m n}=\rho_{m} \rho_{n} \leqslant \alpha \beta$ and so $\alpha \beta \in H_{\rho}$. Also $I_{X} \in H_{\rho}$ since $\rho_{1_{M}} \leqslant I_{X}$. Thus $H_{\rho}$ is a cancellative monoid, and if $M$ is finite, then $H_{\rho}$ is a group.

Similarly, $H_{\lambda}$ is a cancellative monoid which is a group if $M$ is finite.

Next, we define relational morphisms $\tau_{\rho}: M \leftrightarrow H_{\rho}$ and $\tau_{\lambda}: M \rightarrow H_{\lambda}$ by

$$
m \tau_{\rho}=\left\{\alpha \in H_{\rho}: \rho_{m} \leqslant \alpha\right\} \quad \text { and } m \tau_{\lambda}=\left\{\alpha \in H_{\lambda}: \lambda_{m} \leqslant \alpha\right\} .
$$

We show that $\tau_{\rho}$ is a left proper relational morphism of left ample monoids. We have already observed that for each $m \in M$ there is a unit $\alpha$ of $\mathcal{I}(X)$ with $\rho_{m} \leqslant \alpha$ so that $\alpha \in m \tau_{\rho}$ and $m \tau_{\rho}$ is not empty. If $e \in E(M)$, then $\rho_{e}$ is idempotent in $\mathcal{I}(X)$, so $I_{X} \in e \tau_{\rho}$ since $I_{X}$ is the greatest idempotent in $\mathcal{I}(X)$. In particular, $I_{X} \in 1_{M} \tau_{\rho}$, and $\left(m \tau_{\rho}\right)^{+}=\left\{I_{X}\right\} \subseteq m^{+} \tau_{\rho}$ for all $m \in M$.

Let $m, n \in M, \alpha \in m \tau_{\rho}$ and $\beta \in n \tau_{\rho}$. Then $\rho_{m} \leqslant \alpha$ and $\rho_{n} \leqslant \beta$, and, as above, $\rho_{m n} \leqslant \alpha \beta$ so that $\alpha \beta \in(m n) \tau_{\rho}$. Thus $\left(m \tau_{\rho}\right)\left(n \tau_{\rho}\right) \subseteq(m n) \tau_{\rho}$. 
Finally, suppose that $m, n \in M$ are such that $m \tau_{\rho} \cap n \tau_{\rho} \neq \emptyset$. Let $\alpha \in m \tau_{\rho} \cap n \tau_{\rho}$, so that $\rho_{m} \leqslant \alpha$ and $\rho_{n} \leqslant \alpha$. Then we have $\rho_{m}=\rho_{m} \rho_{m}^{-1} \alpha=\rho_{m}^{+} \alpha$ and $\rho_{n}=\rho_{n} \rho_{n}^{-1} \alpha=\rho_{n}^{+} \alpha$. Using the fact that $\rho$ is a $(\cdot)^{+}$-embedding, we have

$$
\rho_{m^{+} n}=\rho_{m^{+}} \rho_{n}=\rho_{m}^{+} \rho_{n}^{+} \alpha=\rho_{n}^{+} \rho_{m}^{+} \alpha=\rho_{n}^{+} \rho_{m}=\rho_{n^{+} m},
$$

so that $m^{+} n=n^{+} m$ since $\rho$ is injective.

In an exactly similar way, $\tau_{\lambda}$ is a right proper relational morphism of right ample monoids.

We now consider the $\left(H_{\rho}, H_{\lambda}\right)$-category $\mathcal{X}=\mathcal{X}_{\left(\tau_{\rho}, \tau_{\lambda}\right)}$ associated with the relational morphisms $\tau_{\rho}$ and $\tau_{\lambda}$. We know that this is a proper ample category, and that the associated monoid $C_{(1,1)}$ is a proper ample monoid. In fact, $C_{(1,1)}$ is a proper ample cover of $M$.

Define the map $\theta: C_{(1,1)} \rightarrow M$ by

$$
(b,((1, b), m,(a, 1)), a) \theta=m .
$$

It is clear that $\theta$ is onto since, for any $m \in M$, there exist $a \in m \tau_{\rho}$ and $b \in m \tau_{\lambda}$ and so

$$
p=((1, b), m,(a, 1)) \in \operatorname{Mor} \mathcal{X}, \quad(b, p, a) \in C_{(1,1)} \quad \text { and } \quad(b, p, a) \theta=m .
$$

Also $\theta$ maps the identity of $C_{(1,1)}$ to the identity of $M$.

Let $\gamma_{1}=\left(b_{1},\left(\left(1, b_{1}\right), m_{1},\left(a_{1}, 1\right)\right), a_{1}\right)$ and $\gamma_{2}=\left(b_{2},\left(\left(1, b_{2}\right), m_{2},\left(a_{2}, 1\right)\right), a_{2}\right)$ be arbitrary elements of $C_{(1,1)}$. Then

$$
\gamma_{1} \gamma_{2}=\left(b_{1} b_{2},\left(\left(1, b_{1} b_{2}\right), m_{1} m_{2},\left(a_{1} a_{2}, 1\right)\right), a_{1} a_{2}\right)
$$

and so

$$
\left(\gamma_{1} \gamma_{2}\right) \theta=m_{1} m_{2}=\left(\gamma_{1} \theta\right)\left(\gamma_{2} \theta\right)
$$

Now

$$
\gamma_{1}^{+} \theta=\left(1,\left((1,1), m_{1}^{+},(1,1)\right), 1\right) \theta=m_{1}^{+}=\left(\gamma_{1} \theta\right)^{+}
$$

and similarly

$$
\gamma_{1}^{*} \theta=\left(\gamma_{1} \theta\right)^{*}
$$

It remains to show that $\theta$ separates idempotents. But this is clear, since

$$
E\left(C_{(1,1)}\right)=\{(1,((1,1), e,(1,1)), 1): e \in E(M)\} .
$$

Therefore, $C_{(1,1)}$ is a proper ample cover of $M$.

To conclude, we observe that if $M$ is finite, then certainly $H_{\rho}$ and $H_{\lambda}$ are finite, and hence $C_{(1,1)}$ is finite.

We briefly consider semigroups as opposed to monoids. It is clear how to extend the definitions of ample, proper and proper cover from monoids to semigroups. Thus, if $S$ is an ample semigroup, we say that a proper ample semigroup $\hat{S}$ is a proper cover of $S$ if there is a $(2,1,1)$-morphism $\theta$ from $\hat{S}$ onto $S$ that separates idempotents. 
Corollary 4.3. Every ample semigroup has a proper ample cover.

Proof. Let $S$ be an ample semigroup. The monoid $S^{1}$ is also ample and by the last theorem it has a proper cover $T$ by means of a $(2,1,1,0)$-morphism $\theta: T \rightarrow S^{1}$. We can show that $S \theta^{-1}$ is a $(2,1,1)$-subalgebra of $T$ and so it is also a proper ample semigroup and it is a cover of $S$.

Acknowledgements. This work was partially supported by 'Financiamento Programático of CAUL' and project POCTI 'Fundamental and Applied Algebra' of FCT and FEDER.

\section{References}

1. A. El Qallali And J. Fountain, Proper covers for left ample semigroups, Semigroup Forum, in press.

2. J. Fountain, A class of right PP monoids, Q. J. Math. 28 (1977), 285-300.

3. J. Fountain and G. M. S. Gomes, Left proper E-dense monoids, J. Pure Appl. Alg. 80 (1992), 1-27.

4. J. Fountain and G. M. S. Gomes, Proper left type-A monoids revisited, Glasgow Math. J. 35 (1993), 293-306.

5. J. Fountain and G. M. S. Gomes, Finite abundant semigroups in which the idempotents form a subsemigroup, preprint (2002), available at http://www-users.york.ac.uk/ $\sim$ jbf1/avg.pdf.

6. J. M. HowIE, Fundamentals of semigroup theory (Oxford University Press, 1995).

7. M. V. Lawson, The structure of type A semigroups, Q. J. Math. 37 (1986), 279-298.

8. M. V. LAWson, Inverse semigroups (World Scientific, 1998).

9. D. B. MCALISTER, One-to-one partial right translations of a right cancellative semigroup, J. Alg. 43 (1976), 231-251.

10. S. W. Margolis and J.-E. Pin, Inverse semigroups and extensions of groups by semilattices, J. Alg. 110 (1987), 277-297.

11. F. PASTIJn, A representative of a semigroup by a semigroup of matrices over a group with zero, Semigroup Forum 10 (1975), 238-239.

12. C. P. Simmons, Small category theory applied to semigroups and monoids, PhD thesis, University of York (2001). 